\title{
Improved Echo cancellation in VOIP
}

\author{
Patrashiya Magdolina Halder \\ Dept. of Electronics and Telecommunication Engineering \\ Daffodil International University \\ Dhaka, Bangladesh
}

\begin{abstract}
VoIP (voice over internet protocol) is very popular communication technology of this century and has played tremendous role in communication system. It is preferred by all because it deploys many benefits it uses Internet protocol (IP) networks to deliver multimedia information such as speech over a data network. VoIP system can be configured in these connection modes respectively; $\mathrm{PC}$ to $\mathrm{PC}$, Telephony to Telephony and $\mathrm{PC}$ to Telephony. Echo is very annoying problem which occurs in VoIP and echo reduces the voice quality of VoIP. It is not possible to remove echo $100 \%$ from echoed signal because if echo is tried to be eliminated completely then the attempt may distort the main signal. That is why echo cannot be eliminated echo perfectly but the echo to a tolerable range. Clipping is not a good solution to suppress echo because part of speech may erroneously removed. Besides an NLP does not respond rapidly enough and also confuses the fading of the voice level at the end of a sentence with a residual echo. This paper has proposed echo cancellation in VoIP that has been tested and verified by MATLAB. The goal was to suppress echo without clipping and distorting the main signal. By the help of MATLAB program the echo is minimized to enduring level so that the received signal seems echo free. The percentage of suppressing echo varies with the amplitude of the main signal. With regarding the amplitude variation in received (echo free) signal the proposed method performs better in finding the echo free signal than the other conventional system.
\end{abstract}

Keywords- PSTN; round trip delay; impedance; inverse filtering; denoise; histogram amplifier; repeater.

\section{INTRODUCTION}

Around 20 years of research on VoIP, some problems of VoIP are still remaining and a substantial problem in telecommunications is the generation of echo. Echo is a phenomenon where a delayed and distorted version of an original signal is reflected back to the source. Echo is a congenital problem which mainly occurs in PSTN (Public Switching Telephone Network) [1]. Echo occurs in analogy part of a telecommunication system .Echo is generated by human voice is heard as they are reflected from the floor, walls and other neighboring objects. If a reflected wave arrives after a very short time of direct sound, it is considered as a spectral distortion or reverberation. However, when the leading edge of the reflected wave appears again a few tens of milliseconds after the direct sound then it is heard as an audible echo [2]. Echo is annoying when the round trip delay exceeds $30 \mathrm{~ms}$. such an echo is typically heard as a hollow sound. Echoes must be loud enough to be heard. Echo which is less than thirty $30 \mathrm{~dB}$ is rarely to be noticed. But when

\author{
A.K.M. Fazlul Haque \\ Dept. of Electronics and Telecommunication Engineering \\ Daffodil International University \\ Dhaka, Bangladesh
}

round trip delay exceeds $30 \mathrm{~ms}$ and echo strength exceeds 30 $\mathrm{dB}$, echoes become steadily more disruptive. Every echo does not reduce voice quality. There are mainly two kinds of echo, that is Hybrid echo and Acoustic echo. Hybrid echo, line echo or electrical echo is different names given to the echo generated by an impedance mismatch in the analog local loop. The impedance mismatch occurs when the two-wire network meets the four-wire network [3]. The impedance of subscriber lines vary from one subscriber to the next, this time sharing makes it impossible to provide a perfect impedance match for every line [4]. Acoustic echo is caused by acoustic coupling problems between a telephone's speaker and its microphone. Acoustic echo can occur in mobile phones, wire line telephones or in a hands-free set of a speaker phone [4]. It can be caused by hand-set crosstalk in poor quality handsets or by echo in the environment surrounding the caller. There are some works on frequency reuse scheme [3, 6-9].

According to asterisk echo cancellation previously called carbon profile $[6,7]$ is operated by generating multiple copies of the received signal, each delayed by some small time increment. These delayed copies are then scaled and subtracted from the original received signal.Srinivasaprasath Raghavendran et al [3] has proposed an echo cancellation process using MATLAB but there the far end signal and the near end signal is taken separately and then tested whether there is echo or not by Double talk detector. This process also includes NLMS and subtraction .Ganesan Periakarruppan, Andy L.Y.Low, Hairul Azhar b Abdul Rashid et al [8] introduced that PBEC the sample to generate the echo replica model will be used to subtract the. Jerker Taudien el al [9] suggested Line probing is a method of inserting a known signal at the far-end and recording the near-end signal. The two signals are then analyzed together for various impediments. Three tone sweeps of different power levels are used to probe the line in the non-linear distortion analysis tool. The tone sweeps are recorded in three different power levels to detect clipping.

All these procedures require more things than this proposed method. This paper has suggested cancelling echo from echoed signal. Only received signal is analysed here so it is not needed to analyse near end and far end signal separately. Besides subtraction and clipping is not required here which ma affect the main signal. This is a very simple program which eliminates echo. Inverse filtering is used here which analyse the received signal and remove echo from the acquired signal. 


\section{BACKGROUND}

Within the caller's telephone, a certain amount of the signal from the microphone is fed straight back to the earpiece. An improperly balanced hybrid won't correctly filter out the entire transmitted signal, and will reflect some of it back down the other half of the trunk. Imbalance may be from poor design (common) or unpredictable. The reasons of echo are as follows:

1. Poor room acoustics

2. Marginal microphones for soft terminals

3. Low quality cellular handsets

4. Deficient echo control in the terminal device its

5. Bridge-taps (something done by the Telco, seldom seen any more)

6. Use of lengthy untwisted wire within the subscriber's premises

\section{Simulation AND Results}

This program is to explore the problem of echo cancellation. Iinverse filtering (IF) is a widely known method for voice and speech analysis, which mainly works on estimating the source of voiced speech. This method enables to estimate the glottal volume velocity waveform or glottal airflow. The idea behind inverse filtering is to form a computational model for glottal pulse detection by filtering the speech signal.

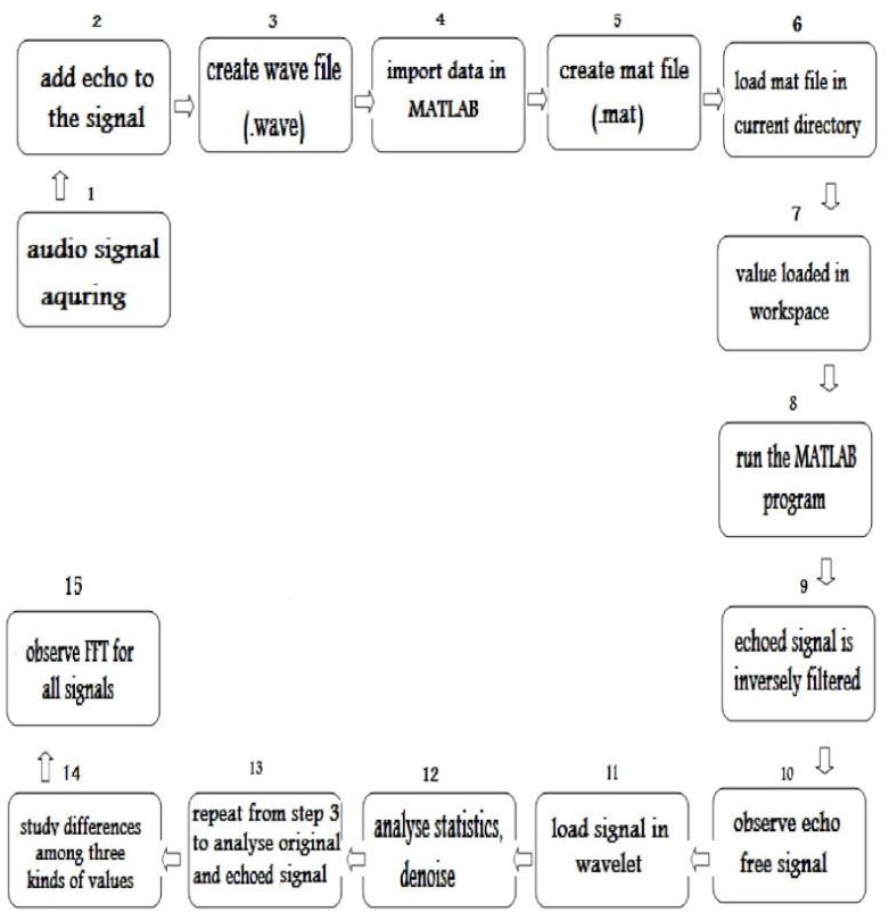

Figure 1. Flowchart for echo cancellation using MATLAB

This flowchart is given bellow for echo suppression using
MATLAB (Fig. 1) .The main signal, signal with echo and signal without echo is showed respectively in Fig. 2, 3 and 4. This signal can be denoised using Matlab to remove echo from this signal.

For the experiment at first voice signal is acquired, it can be done by any kind of speech recorder. Then this acquired voice signal is used to create a .wave file using the audio signal. After that Matlab software is opened the data of the signal is imported to create .mat file. This mat file has to be loaded to transfer the value of the speech signal in workspace. To remove echo from the signal wavelet is used. To inspect the signals by wavelet wave menu is typed in the command window of Matlab; a new window of wavelet will be displayed then. The echoed signal is loaded in wavelet and then the signal is analyze, view the statistics, denoise and analyze the signal. Doing all these signal with echo can be analyzed. The signals can be compressed to get better result
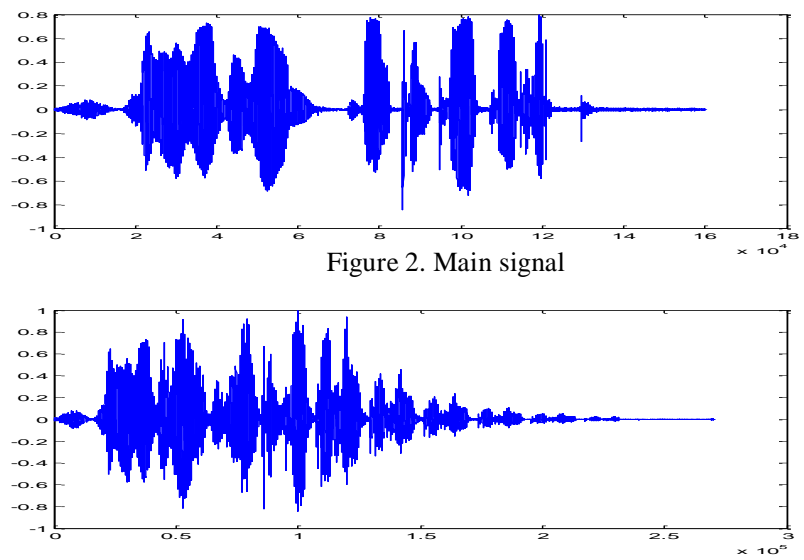

Figure 3. Signal with echo

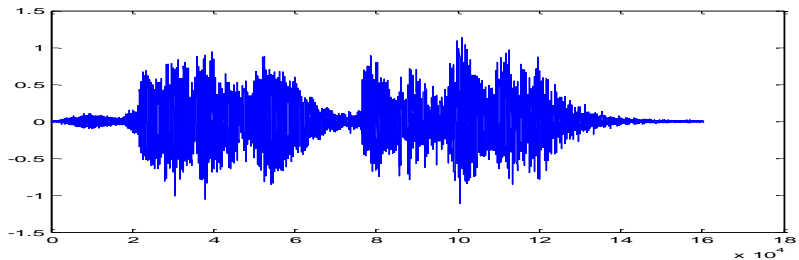

Figure 4. Signal without echo

By running the program, hearing the signal it will be clear that echo is removed from the echoed signal and the quality of signal is improved. The signal is clear and each part of speech is present. If the simulation figures are observed closely then it can be seen that the wave of main signal is like overlapped in the echoed signals figure. The refined signal is almost look alike the echo free signal, there is just change in the amplitude of the signal. So it can be said that the improved signal and the outcome signal both prove that the attempt is successful to remove echo and perform better.

Histogram is used to analyze the speech signal which showed in Fig. 5, 6 and 7. 


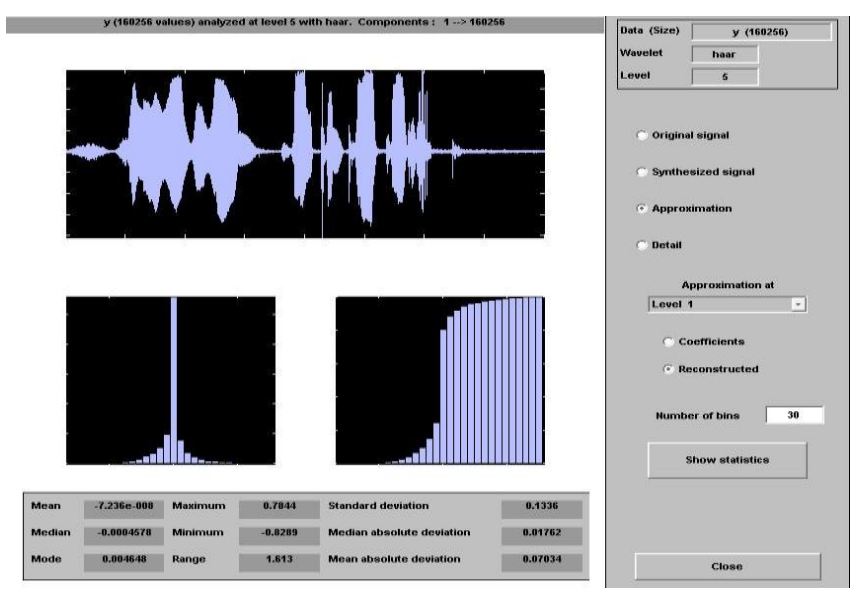

Figure 5. Analyzing main signal with wavelet

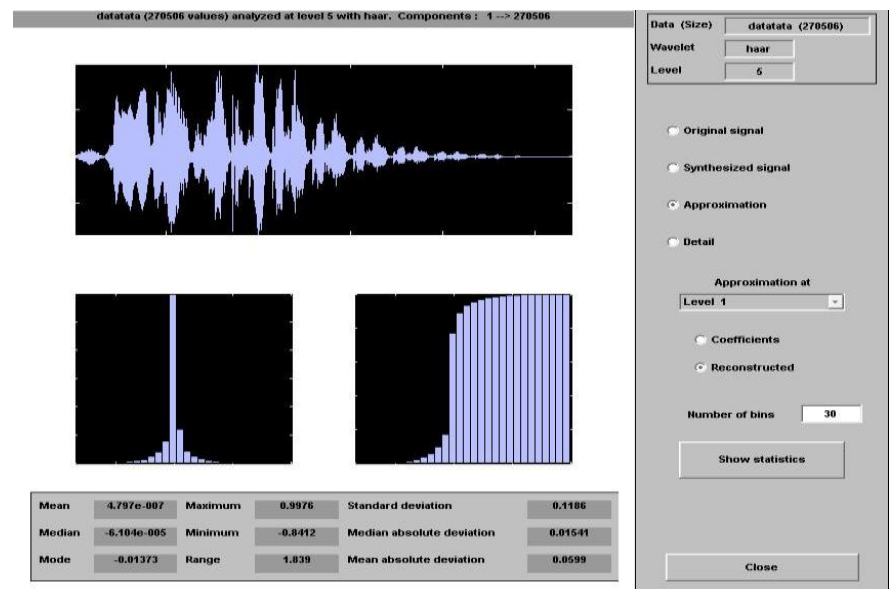

Figure 6. Analyzing signal with echo with wavelet

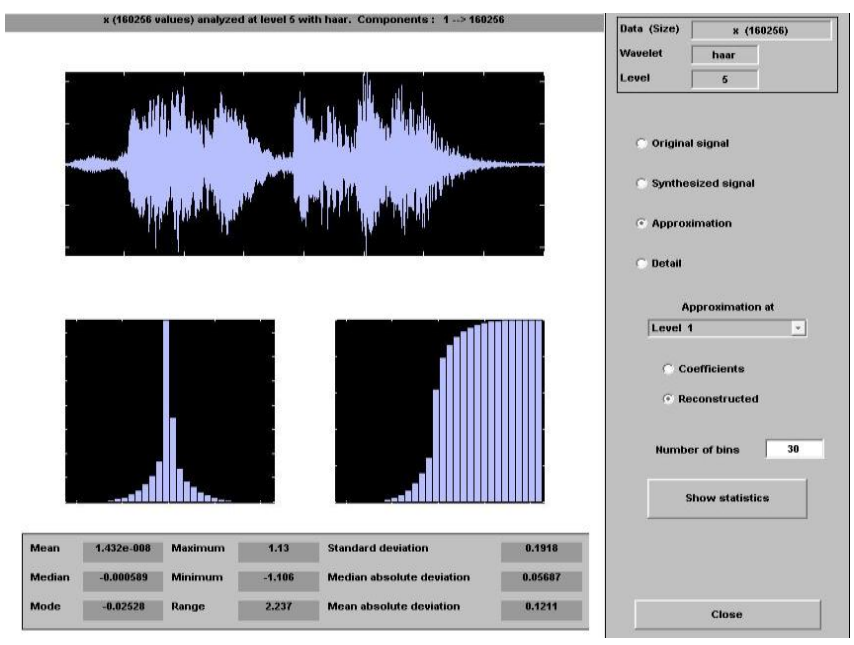

Figure 7. Analyzing signal without echo with wavelet

The following figure 8 shows the difference among the signals with the help of FFT (Fast Fourier Transform)

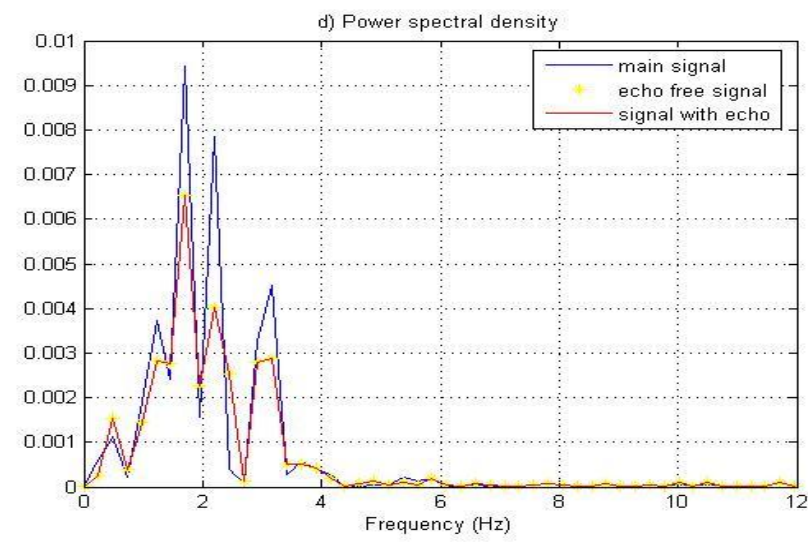

Figure 8. Analyzing all signals using FFT

Difference between signal with echo and signal without echo is observed. By seeing the waveforms generally it can be said that echo is removed. There is difference in amplitude among the signals; the amplitude can be regained by using amplifier or repeater.

For further analyzing different statistical values are extracted from this experiment and taking the values a table 4.4 and a graph (Fig. 9) is plotted, this will help to prove that echo is removes from the echoed signal.

TABLE I. SIGNAL ANALYZING

\begin{tabular}{|l|l|l|l|l|l|l|}
\hline \multicolumn{2}{|l|}{ Main signal } & \multicolumn{2}{l|}{ Echoed signal } & \multicolumn{2}{l|}{$\begin{array}{l}\text { Signal without } \\
\text { echo }\end{array}$} \\
\hline Mean & $\begin{array}{l}-7.236 \\
\mathrm{e}-008\end{array}$ & 0.7844 & $\begin{array}{l}4.797 \mathrm{e}- \\
007\end{array}$ & 0.999 & $\begin{array}{l}1.432 \mathrm{e}- \\
008\end{array}$ & 1.13 \\
\hline Median & - & -0.8289 & $-6.104 \mathrm{e}-$ & - & - & - \\
& 0.0004 & & 005 & 0.841 & 0.000589 & $\begin{array}{l}1.10 \\
6\end{array}$ \\
\hline $\begin{array}{l}\text { Mode } \\
\text { Standard } \\
\text { deviation }\end{array}$ & 0.0046 & 1.613 & -0.01373 & 1.839 & -0.02528 & $\begin{array}{l}2.23 \\
7\end{array}$ \\
\hline $\begin{array}{l}\text { Median } \\
\text { absolute } \\
\text { deviation }\end{array}$ & 0.01762 & & & & & \\
\hline $\begin{array}{l}\text { Mean } \\
\text { absolute } \\
\text { deviation }\end{array}$ & 0.07034 & 0.1186 & & & & \\
\hline
\end{tabular}




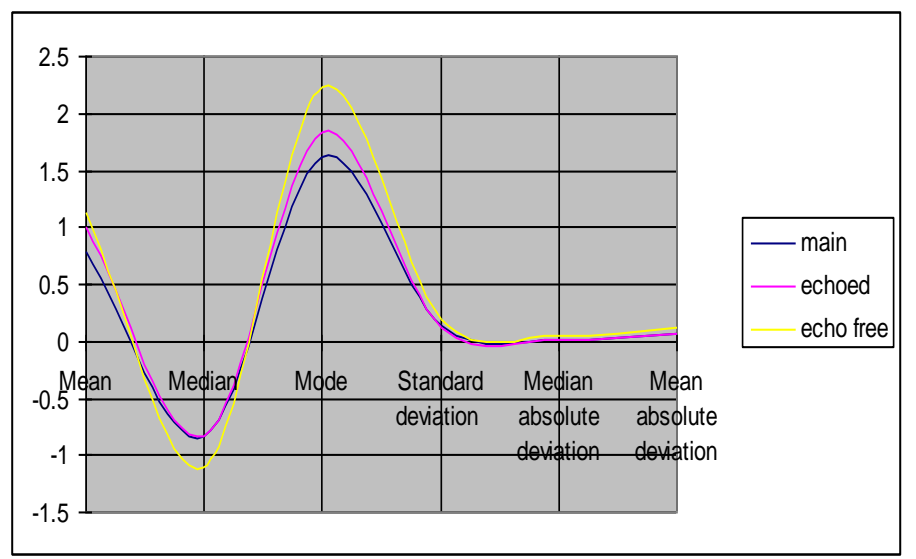

Figure 9: Comparing main signal, echoed signal and signal without echo.

Difference between the signal without echo and signal with echo is observed. The mean, median, mode all the values are analyzed more over the compressed signal is almost matched with the desire echo free signal, which is our goal.

\section{CONCLUSION}

In this report, empirical audio signal has been considered to evaluate the performance of echo cancellation. It has been observed that the echo is suppressed without changing or distorting the main signal and user of VOIP can hear clear sounds. This program is easy to use and simpler than the conventional methods of suppressing echo. The simulation results have been tested and verified using Wavelet Tool. Power spectrum density has also been used to observe the difference of the received signal. The proposed system has found better performance in finding the echo cancellation than the conventional methods which can be adopted to suppress echo and take the fullest advantage of VOIP telephony.

\section{REFERENCES}

[1] A Survey on Voice over IP over Wireless LANs Haniyeh Kazemitabar, Sameha Ahmed, Kashif Nisar, Abas B Said, Halabi B Hasbullah

[2] Echo in Voice over IP Systems Series VoIP Performance Management Date January 2006

[3] Implementation of an Acoustic Echo Canceller Using Matlab by Srinivasaprasath Raghavendran College of Engineering University of South Florida October 15, 2003

[4] IP Telephony: Packet-based multimedia communication system By Olivier Hersent, David Gurle, Jean-Pierre Petit

[5] Design a high-performance echo canceller for VOIP application, by Dr. Chang Y. Chob

[6] http://www.voipinfo.org/wiki/view/Asterisk+echo+cancellation

[7] http://www.eetimes.com/design/signal-processing-dsp/4017606/HighPerformance-echo-canceller-for-Asterisk-VoIP-systems

[8] PACKET BASED ECHO CANCELLATION FOR VOICE OVER INTERNET PROTOCOL SIMULATED WITH VARIABLE AMOUNT OF NETWORK DELAY TIME by Ganesan Periakarruppan, Andy L.Y.Low, Hairul Azhar b Abdul Rashid

[9] LINE PROBING IN VOIP NETWORKS TO FIND PERFORMANCE LIMIT OF ECHO CANCELLER by Jerker Taudien, 2007 